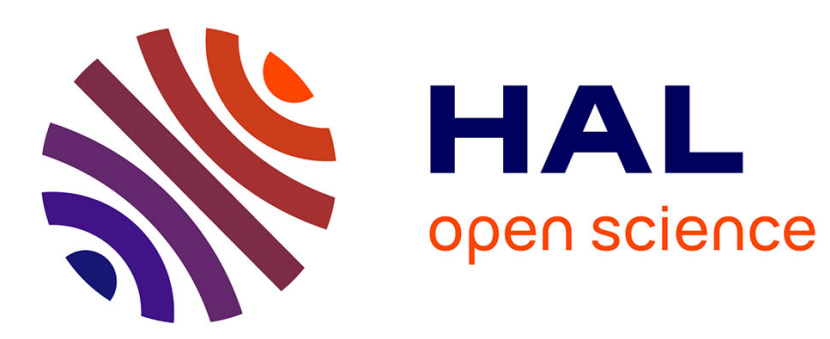

\title{
RNA Splicing, Cell Signaling and Response to Therapies
} Cherine Abou Faycal, Sylvie Gazzeri, Béatrice Eymin

\section{To cite this version:}

Cherine Abou Faycal, Sylvie Gazzeri, Béatrice Eymin. RNA Splicing, Cell Signaling and Response to Therapies. Current Opinion in Oncology, 2016, 10.1097/CCO.0000000000000254 . inserm-02337520

\section{HAL Id: inserm-02337520 https://www.hal.inserm.fr/inserm-02337520}

Submitted on 29 Oct 2019

HAL is a multi-disciplinary open access archive for the deposit and dissemination of scientific research documents, whether they are published or not. The documents may come from teaching and research institutions in France or abroad, or from public or private research centers.
L'archive ouverte pluridisciplinaire HAL, est destinée au dépôt et à la diffusion de documents scientifiques de niveau recherche, publiés ou non, émanant des établissements d'enseignement et de recherche français ou étrangers, des laboratoires publics ou privés. 


\section{RNA Splicing, Cell Signaling and Response to Therapies}

Cherine Abou Faycal ${ }^{1,2}$, Sylvie Gazzeri ${ }^{1,2}$ and Beatrice Eymin ${ }^{1,2 *}$

${ }^{1}$ INSERM, U823, Equipe 2, Bases Moléculaires de la Progression des Cancers du Poumon, Grenoble, 38042, France.

${ }^{2}$ Université Joseph Fourier, Institut Albert Bonniot, Grenoble, 38041, France.

* Corresponding author:

Dr. Beatrice Eymin

INSERM U823 Equipe 2

Institut Albert Bonniot, BP170

38042 Grenoble Cedex 09, FRANCE

Tel: +33476549476/ Fax: +33476549413

email: Beatrice.Eymin@ujf-grenoble.fr

To whom requests for reprints should be addressed 


\section{Abstract}

\section{Purpose of review}

Pre-mRNA alternative splicing is more a rule than an exception as it affects more than $90 \%$ of multi-exons genes and plays a key role in proteome diversity. Here, we discuss some recent papers published in the extensively growing field linking RNA splicing and cancer.

\section{Recent findings}

These last years, the development of high-throughput studies together with appropriate bioinformatic tools have led to the identification of new cancer-specific splicing patterns that allow to distinguish various cancer types and provide new prognosis biomarkers. In addition, the functional consequences of hot spot mutations affecting various components of the spliceosome machinery in cancers have been described. As an example, mis-splicing of the EZH2 histone methyltransferase pre-mRNA in response to hot spot mutation of the splicing factor SRSF2 was found to participate to the pathogenesis of myelodysplastic syndrome. Moreover, proofs of principle that targeting the RNA splicing machinery can be used to correct aberrant mis-splicing, kill oncogene-driven cancer cells or reverse resistance of tumor cells to targeted therapies have been done. As another example, the core spliceosomal function was recently found to be critical for the survival of Myc-driven breast cancer cells, rendering them hypersensitive to spliceosome inhibitors.

\section{Summary}

Dysregulation of pre-mRNA alternative splicing appears to be one of the hallmarks of cancer. The characterization of novel splicing signatures in cancer as well as the identification of original signaling networks involving RNA splicing regulators should allow to decipher novel oncogenic mechanisms and to developp new therapeutic strategies. 


\section{Keywords}

Alternative pre-mRNA splicing, Cancer, Cell signaling, Therapies. 


\section{Introduction}

In eukaryotes, gene expression is finely controlled by complex regulatory processes that affect all steps of RNA expression, extending from transcription to maturation in the nucleus, mRNA cytosolic export, stability and translation. One of the crucial step inside this network is the pre-mRNA constitutive splicing process during which intronic sequences are removed from the pre-mRNA and exonic sequences joined to form the mature mRNA. Another level of regulation during this process is represented by alternative splicing (AS). Through the use of alternative promoters, alternative polyadenylation sites, retained introns or alternatively spliced exons (exon cassette or mutually exclusive exon), AS leads to the generation of several coding and non-coding mRNA variants from the same gene. Therefore, one of the main consequences of AS is to remodel the proteome through the synthesis of various protein isoforms displaying different biological activities. This process is tightly controlled across different tissues and developmental stages, and dysregulation of AS is closely associated with various human diseases including cancer. The identification of the alternative splicing isoforms expressed in tumors is therefore of utmost relevance to unravel novel oncogenic mechanisms and for the development of new therapeutic strategies. In this review, we discuss some recently published studies in this extensively growing field linking RNA splicing and cancer. 


\section{Dysregulation of alternative splicing: one of the hallmarks of cancer}

This last decade, the development of high-throughput studies as well as the improvement of bioinformatic tools have extensively increased the amount of expression data regarding splice variants in cancer, and unraveled the existence of cancer-specific splicing patterns that contribute likely to tumor progression through modulation of every aspect of cancer cell biology $[1,2]$. Accordingly, by analyzing RNA-Seq databases from the Cancer Genome Atlas project, three studies published this year characterized novel splicing signatures in different cancer types $\left[3^{*}, 4^{*}, 5\right]$. Many of these cancer-associated splicing events were functionally associated with cell cycle control, cytoskeletal organization, migration, cell-cell adhesion and more unexpectedly insulin signaling pathway. Importantly, these splicing events allowed to separate cancer from normal tissues, to distinguish distinct cancer types (ie breast carcinoma versus lung cancer) or histological subtypes within the same cancer type (ie lung adenocarcinoma versus squamous lung carcinoma), as well as to predict patient survival. As an example, Sebestyen and colleagues analyzed more than 4000 samples in nine different cancer types and found specific splicing signatures for basal-like breast tumors involving the tumor driver CTNND1 [4*]. They also identified 244 isoform switches for which the relative expression change occurs in the most abundant isoform, suggesting that they may have a functional impact. Importantly, most of these switches were independent of somatic mutations, indicating that alterations in chromatin structure or aberrant expression of splicing factors could explain these splicing events. Consistently, deregulation of specific splicing regulators including RBFOX2, MBNL1/2 and QKI proteins was further found to account for hundred of cassette exons for which splicing was altered in multiple cancer types $\left[3^{*}\right]$, highlighting these specific factors as critical regulators of cancer-specific AS. Overall, and although many splice variants might not be detected owing to their low abundance, these large scale studies unraveled a huge amount of cancer-altered splice events in distinct cancer 
types. Deepened investigations are now required in order to identify the functional consequences of these splicing events as well as the regulatory upstream signals that control them. Such studies are mandatory if we want to select those AS events that really contribute to the carcinogenesis process and are therefore prime targets for therapeutic purpose. As an example, exon array studies performed in Non Small Cell Lung Cancers (NSCLC) have identified most prevalent tumor-associated changes in transcripts from genes which regulate angiogenesis, actin-cytoskeleton remodeling and WNT/NOTCH signaling, namely Vascular Endothelial Growth Factor-A (VEGF-A), Microtubule-Actin Cross-linking Factor 1 (MACF1), Amyloid Beta (A4) Precursor Protein (APP), and the Numb (Drosophila) homolog (NUMB) [6]. QKI is frequently down-regulated in lung cancer and its down-regulation is significantly associated with a poorer prognosis. Consistently, QKI was later found to inhibit lung cancer cells growth at least in part through the regulation of the alternative splicing of NUMB $\left[7^{*}\right]$. These data are thus consistent with the fact that only a restricted number of splicing factors and AS events could be critical for cancer progression but distinct according to the tumor type. In addition, splicing regulators may play other functions than regulating « classical» AS. circRNAs result from a non-canonical form of alternative splicing most commonly where the splice donor site of one exon is ligated to the splice acceptor site of an upstream exon. As an example, the role of QKI in the production of hundred of these circRNAs was demonstrated during TGF- $\beta$ induced Epithelial to Mesenchymal Transition (EMT) $\left[8^{* *}\right]$. As EMT is involved in cancer metastasis, one may postulate that production of « misspliced » circRNAs also contributes to tumor progression.

\section{Functional consequences of mutations affecting RNA splicing regulators}

The splicing process is carried out by the spliceosome, a complex macromolecular machinery constituted of five small nuclear ribonucleoprotein particles (U1, U2, U4, U5 and U6 
snRNPs) and more than 200 ancillary proteins. Up to now, a growing number of studies have demonstrated abnormal expression (either downregulation or overexpression) of some spliceosome components in cancers. More recently, somatic mutations of splicing factors were identified in myelodysplastic syndrome (MDS) [9], acute myeloid leukemia (AML; The Cancer Genome Atlas) and lung adenocarcinoma (LUC) [10]. Importantly, they were mutually exclusive, suggesting that spliceosome mutations may have the same functional effect on oncogenesis. Until these last 18 months, it was poorly known how these mutations contribute to cancers. Hotspot mutations in SF3B1 at a residue lysine in position 700 occur in $16 \%$ and $6 \%$ of papillary and mucinous breast carcinoma [11]. Interestingly, SF3B1 mutation was associated with differentially spliced events in TMEM14C, RPL31, DYNL11 or ABCC5 genes and more importantly SF3B1 mutant cell lines were found to be more sensitive to spliceostatin A, a SF3B complex inhibitor [11]. U2AF1, a 3' splice site recognition factor, has hotspot mutation at amino acid position 34 in AML, MDS and LUC. Using TCGA databases, Brooks and colleagues identified 131 and 369 splicing alterations significantly associated with U2AF1 mutation in LUC and AML respectively, with 30 splicing alterations being present in both cancer types including three genes of the Cancer Gene Cesus, CTNNB1, CHCHD7 and PICALM [12]. They also demonstrated that mutation of U2AF1 alters its binding to the 3 ' splice site sequence and causes preference for CAG over UAG. More recently, Shirai and colleagues demonstrated that transgenic mice expressing mutant $\mathrm{U} 2 \mathrm{AF} 1(\mathrm{~S} 34 \mathrm{~F})$ in bone marrow cells display altered hematopoiesis and changes in pre-mRNA splicing in hematopoietic progenitor cells affecting mainly RNA processing genes, ribosomal genes, and recurrently mutated MDS- and AML-associated genes [13**]. Interestingly, both monocytes and B cells were reduced in $\mathrm{U} 2 \mathrm{AF} 1(\mathrm{~S} 34 \mathrm{~F})$ mutated mice and evidenced increased phospho-H2AX staining, suggesting that U2AF1(S34F) mutation may have consequences for genome stability, which is reminiscent with mis-splicing being involved in the co- 
transcriptional formation of R-loops (RNA:DNA hybrid) leading to DNA double strand breaks. The SRSF2 protein is a member of the serine/arginine-rich (SR) protein family that contributes to both constitutive and alternative splicing. SRSF2 mutations on its P95 proline residue occur in 20-30\% MDS patients and are associated with adverse outcome [9]. Consistent with the critical role of SRSF2 mutation in MDS, hematopoietic-specific conditional Srsf2 knock-in mice, heterozygous for SRSF2-P95H mutation, developed myelodysplasia while Srsf2-deficient mice did not [14**]. This phenotype was associated with global alterations of gene expression affecting several hematopoietic regulators including Gata1, Gata2, Cdkn1a and Hoxb2. In addition, proline 95 mutations were found to change the RNA binding specificity of SRSF2, thereby promoting the inclusion of a highly conserved «poison» cassette exon of EZH2 (Enhancer of Zeste Homolog 2), a histone methyltransferase belonging to the Polycomb repressive complex 2, leading to nonsensemediated decay. This SRSF2(P95H)-induced missplicing event could be relevant for MDS pathogenesis as restoring EZH2 expression partially rescues hematopoiesis in SRSF2 mutant cells [14**]. Moreover, dysregulated splicing of U12-type intron in MDS was also associated with mutations of ZRSR2, an essential component of the minor spliceosome assembly [15]. Later on, germline and somatic mutations in the gene encoding for the DEAD-Box RNA helicase DDX41 were identified in myeloid neoplasms and were again mutually exclusive with mutations of other spliceosomal proteins $\left[16^{* *}\right]$. These mutations were shown to alter DDX41 interaction with major components of U2 (SF3B1) and U5 (PRPF8) spliceosomes and to lead to the missplicing of genes such as ZMYM2, a zinc finger protein involved in a histone deacetylase complex. Overall, these studies clearly evidence the role of splicing regulators mutation in triggering missplicing patterns in cancers (Figure 1). In addition, they highlight some epigenetic regulators as potential target genes of these mutated proteins. Although these mutations appear to be mutually exclusive, both mutation and deregulated 
expression (downregulation or overexpression) of distinct splicing factors co-occur in the same tumors. Further functional studies and mouse models are now needed to decipher whether and how these anomalies cooperate during the tumorigenic process.

\section{New insights linking RNA splicing and intra-cellular signaling networks}

Multiple post-translational modifications have been shown to control the expression and/or the activity of splicing factors. However, how the RNA splicing machinery is connected within intra-cellular signaling networks and integrated within physiological stimuli is only an emerging field. Several recent studies provided new data in this setting. As a few examples, an unpreviously known link was identified between iron homeostasis and a SRSF7-dependent control of the alternative splicing of the apoptosis regulator Fas/CD95 [17**]. In addition, hypoxia was reported to increase the level of CLK1, SRPK1 and SRPK2 by a HIF1dependent mechanism leading to SR proteins hyperphosphorylation [18]. More recently, Bordeleau and colleagues demonstrated that matrix stiffness also controls SR proteins hyperphosphorylation and regulates by this way the alternative splicing of fibronectin, PKC $\beta \mathrm{II}$ and VEGF-A [19*]. The mammalian genome is at constant risk of mutation, and accumulation of DNA lesions is intimately linked to cancer. It is therefore crucial for the cell to detect DNA lesions, signal their presence and promote their repair. All these mechanisms are collectively termed the DNA Damage Response (DDR). These last years, numerous crosstalks between DDR and RNA splicing have emerged which unraveled splicing factors as critical contributors of DDR through prevention of R-loops formation or interaction with components of the DNA repair machinery [20]. Modulating the expression and/or activity of RNA splicing factors could therefore offer alternative therapeutic strategies to increase the sensitivity of cancer cells in combination with classical chemotherapies. Three recent studies further deepened this connection. In quiescent cells, Tresini and colleagues demonstrated that 
transcription-blocking DNA lesions caused by UV irradiations deplete chromatin in latematuration stage spliceosomes, namely U2 and U5 snRNPs, by inducing their relocalization. Such displacement leads to R-loops formation and activation of a non-canonical Ataxia Telangiectasia Mutated (ATM) signaling pathway that prevents further spliceosome organization through a forward feedback loop and alters alternative splicing [21**]. In addition, in a transgenic mouse model that mimicks progressive telomere erosion and persistent DNA damage signaling, altered differentiation of common myeloid progenitors and occurrence of a MDS phenotype were associated with the repression of mRNA splicing/processing genes including SRSF2, as well as with aberrant splicing of genes involved in genome stability, DNA repair or chromatin remodeling such as the DNA methyltransferase DNMT3A [22**]. Interestingly, a correlation between activation of the ATR kinase and decreased SRSF2 levels was found in MDS CD34+ patients cells, suggesting that ATR may regulate SRSF2 expression. Overall, these studies reinforce the previously published connection between ATM, ATR and some components of the spliceosome [23]. Another study showed that a major gatekeeper of genome stability, namely BRCA1, interacts with and recruits the mRNA-splicing machinery onto BRCA1/BCLAF1 target genes in response to DNA damage, thereby promoting transcript stability and protein expression. Importantly, the depletion of BRCA1, BCLAF1 or other spliceosome members of this complex resulted in sensitivity to DNA damage and defective DNA repair [24**]. This study provides evidence that key DDR components also contribute to pre-mRNA alternative splicing of specific target genes. Therefore, RNA splicing machinery controls DNA damage machinery and "vice versa". As DDR is a critical gatekeeper against genome instability, such interplay provides an additional mechanism by which aberrant RNA splicing could contribute to tumorigenesis. 


\section{Therapeutic strategies targeting RNA splicing in cancer: proofs of principle}

Aberrant pre-mRNA splicing promotes cell survival in response to chemotherapy through the deregulation of genes involved in apoptosis or alteration in drug metabolism [25]. In addition, modulation of alternative splicing can also account for primary or secondary resistance to targeted therapies as previously demonstrated for the Ikaros splice variant Ik6 that drives resistance to imatinib in BCR-ABL positive ALL cell lines [26], or for the V600E BRAF splice variant BRAF3-9, which results from skipping of BRAF exons 4-8, and correlates with acquired resistance to vemurafinib in melanoma patients [27]. As management of cancerous patients with primary/acquired resistance to targeted therapies remains a significant challenge with therapeutic, social and economic impacts, these data provide the rationale for targeting RNA splicing in cancer. In this setting, it was proposed that spliceosome mutants represent a driver force in some tumors which may thus be sensitive to spliceosome inhibitors. However, as demonstrated very recently, key driver oncogenes such as Myc can also determine the sensitivity to spliceosome inhibitors of tumor cells lacking spliceosome mutations [28]. Therefore, these results pave the way for testing spliceosome inhibitors in a large variety of cancers whatever their spliceosome mutational status. Besides targeting spliceosome components, mRNA Seq approaches recently provided evidence that small molecules inhibiting the SR proteins kinases CLKs and SRPKs favor the production of splice variants with premature stop codon, thereby leading to protein depletion for multiple genes, including those involved in growth and survival pathways such as S6K, EGFR, EIF3D and PARP [29]. Small-molecule inhibitors of SRPK1, namely SPHINX and SRPIN340, were also found to switch the splicing of VEGF-A towards the anti-angiogenic splice variant $\mathrm{VEGF}_{165} \mathrm{~b}$ in prostate cancer cells and to decrease tumor growth in an orthotopic mouse model [30]. However, adding another level of complexity, both oncogenic and tumor suppressive functions of SRPK1 were recently highlighted [31**, 32*]. In addition, in luminal breast 
cancer cells, CLK2 down-regulation was reported to mediate epithelial to mesenchymal transition and splicing of target genes involved in cancer cell invasion and metastasis [33]. Therefore, before targeting SR-phosphorylating kinases for therapeutic purpose, we have to keep in mind that deleterious effects might occur depending on the tumor type and microenvironnment.

At the molecular level, recent pre-clinical studies also demonstrated that sudemycin E, a general spliceosome SF3B inhibitor, binds to SF3B1 and induces its displacement from nucleosome causing a rapid change in alternative pre-mRNA splicing and a G2 arrest, that correlates with a loss of the H3K36me3 modification in chromatin encoding spliced exons [34*]. The recruitment of splicing factors and adaptator proteins through specific histone marks act in a coordinated manner to regulate alternative splicing [35]. H3K36me3 is a modification significantly enriched in actively transcribed gene bodies and around the intronexon boundaries of included exons. Very interestingly, a recent paper demonstrated that AKT1 and AKT3 phosphorylate IWS1, a RNA processing regulator, and allow the recruitment of the histone-methyltransferase SET2, the trimethylation of H3K36 and the splicing of FGFR2 [36**]. Importantly, the authors further demonstrated that IWS1 phosphorylation correlates with AKT phosphorylation and FGFR2 splicing pattern in lung adenocarcinoma patients. This paper provides evidence that direct cross-talks between kinases involved in cell proliferation and/or apoptosis, RNA machinery and chromatin-remodelling enzymes exist to control alternative splicing in tumor. Whether and how spliceosome inhibitors, used or not in combination with other therapies such as chemotherapies, impact on these signaling networks require therefore deepened investigations. Furthermore, in chronic lymphocytic leukemia (CLL) cells, the combination of sudemycin with ibrutinib, a Bruton's tyrosine kinase (BTK) inhibitor, resulted in an enhanced cytotoxic effect involving the 
splicing of IBTK, a physiological inhibitor of BTK [37]. Moreover, spliceostatin A or its analogue meayamycin B (MAMB) was found to limit cell proliferation of vemurafenibresistant cells by decreasing the amount of the BRAF3-9 splice variant, to prevent tumor formation, and to slow-down the growth of vemurafenib-resistant tumors [38**]. As a whole, these studies strongly support the future development of novel strategies targeting the spliceosome machinery to overcome drug resistance (Figure 1). Hence, the first-in-human phase I clinical trial devoid to identify dose-limiting toxicities using the mRNA splicing inhibitor E107 was published two-years ago in advanced solid tumors patients including mainly colorectal or esophageal carcinoma [39].

\section{Conclusion}

These last years, the development of high-throughput technics allowed the identification of a huge number of cancer-related splicing events, together with the discovery of somatic mutations in RNA splicing regulators. These recent insights pave the way for the use of alternative therapeutic strategies designed to target the RNA splicing machinery or to correct specific mis-splicing events in cancer. However, further deepening of the functional consequences of these aberrant splice patterns is needed to identify those events that are critical for tumor progression and/or emergence of resistance to treatments. These events appear largely dependent on the tumor type, adding another layer of complexity.

Word count : 2454 words. 


\section{Key-points}

- New splicing signatures allow to distinguish cancer cells from their normal counterparts, to distinguish distinct tumor types or histological subtypes within the same cancer types as well as to predict patients survival.

- Transgenic mouse models demonstrated that hot spot mutations of various splicing regulators modify their RNA binding specificity leading to mis-splicing events, differentiation defects and development of myelodysplastic syndromes.

- Further insights into the interplay linking the RNA splicing and DNA damage machineries demonstrate that transcription-blocking DNA lesions in non-replicating cells control alternative splicing through chromatin displacement of spliceosome, formation of R-loops and activation of a non-canonical ATM signaling pathway.

- Proofs of principle that targeting RNA splicing machinery can reverse the resistance of cancer cells to targeted therapies have been provided in pre-clinical studies, opening the way for further clinical trials combining drugs targeting RNA splicing together with chemotherapies and/or targeted therapies. 


\section{References}

1. David CJ, Manley JL. Alternative pre-mRNA splicing regulation in cancer: pathways and programs unhinged. Genes Dev 2010; 24:2343-2364.

2. Germann S, Gratadou L, Dutertre M, Auboeuf D. Splicing programs and cancer. J Nucleic Acids 2012; 2012:269570.

*3. Danan-Gotthold M, Golan-Gerstl R, Eisenberg E, et al. Identification of recurrent regulated alternative splicing events across human solid tumors. Nucleic Acids Res 2015; $43: 5130-5144$.

*4. Sebestyen E, Zawisza M, Eyras E. Detection of recurrent alternative splicing switches in tumor samples reveals novel signatures of cancer. Nucleic Acids Res 2015; 43:1345-1356. Based on the RNA-Seq data from the TCGA consortium, these two papers report the first large scale analyses of alternative splicing events occuring in a high number of solid tumors, and identify splicing signatures specific of cancer type or histological sub-type. They provide the rationale for future studies designed for identification of splicing-based diagnostic/prognostic biomarkers and therapeutic targets.

5. Tsai YS, Dominguez D, Gomez SM, et al. Transcriptome-wide identification and study of cancer-specific splicing events across multiple tumors. Oncotarget 2015; 6:68256839.

6. Misquitta-Ali CM, Cheng E, O'Hanlon D, et al. Global profiling and molecular characterization of alternative splicing events misregulated in lung cancer. Mol Cell Biol $2010 ; 31: 138-150$.

*7. Zong FY, Fu X, Wei WJ, et al. The RNA-binding protein QKI suppresses cancerassociated aberrant splicing. PLoS Genet 2014; 10:e1004289. 
This paper highlights a QKI-5/NUMB/NOTCH network as a crucial contributor of lung cancer progression, through the demonstration that QKI-5 down-regulation in these tumors correlates with poor outcome and leads to the expression of a NUMB splice variant containing exon 12 which is able to activate Notch signaling and cell proliferation.

**8. Conn SJ, Pillman KA, Toubia J, et al. The RNA binding protein quaking regulates formation of circRNAs. Cell 2015; 160:1125-1134.

This paper shows that epithelial to mesenchymal transition, which is highly involved during metastatic process, controls the formation of circRNAs resulting from back-splice junctions by a mechanism involving QKI. This study opens the way to the study of QKI-regulated but maybe other RNA splicing factors-regulated circRNAs in cancers.

9. Yoshida K, Sanada M, Shiraishi Y, et al. Frequent pathway mutations of splicing machinery in myelodysplasia. Nature 2011; 478:64-69.

10. Imielinski M, Berger AH, Hammerman PS, et al. Mapping the hallmarks of lung adenocarcinoma with massively parallel sequencing. Cell 2012; 150:1107-1120.

11. Maguire SL, Leonidou A, Wai $\mathrm{P}$, et al. SF3B1 mutations constitute a novel therapeutic target in breast cancer. J Pathol 2015; 235:571-580.

12. Brooks AN, Choi PS, de Waal L, et al. A pan-cancer analysis of transcriptome changes associated with somatic mutations in U2AF1 reveals commonly altered splicing events. PLoS One 2014; 9:e87361.

**13. Shirai CL, Ley JN, White BS, et al. Mutant U2AF1 Expression Alters Hematopoiesis and Pre-mRNA Splicing In Vivo. Cancer Cell 2015; 27:631-643.

**14. Kim E, Ilagan JO, Liang Y, et al. SRSF2 Mutations Contribute to Myelodysplasia by Mutant-Specific Effects on Exon Recognition. Cancer Cell 2015; 27:617-630.

These two papers provide the first functional characterization of two transgenic mouse models displaying either U2AF1(S34F) or SRSF2(P95H) mutation. They highlight the consequences 
of these mutations on normal hematopoiesis as well as their impact on specific pre-mRNA alternative splicing events in link with myelodysplastic development.

15. Madan V, Kanojia D, Li J, et al. Aberrant splicing of U12-type introns is the hallmark of ZRSR2 mutant myelodysplastic syndrome. Nat Commun 2015; 6:6042.

**16. Polprasert C, Schulze I, Sekeres MA, et al. Inherited and Somatic Defects in DDX41 in Myeloid Neoplasms. Cancer Cell 2015; 27:658-670.

This study reports the first germline and somatic mutations of DDX41 in myeloid neoplasms. As mutually exclusive somatic mutations of other RNA helicases genes were also observed, these data lead to the proposal that RNA helicase genes may represent a new class of tumor suppressor genes.

**17. Tejedor JR, Papasaikas P, Valcarcel J. Genome-wide identification of Fas/CD95 alternative splicing regulators reveals links with iron homeostasis. Mol Cell 2015; 57:23-38.

This paper reports the first link between iron homeostasis and regulation of spliceosome machinery, notably through the demonstration that variation in iron levels has a direct effect in the RNA binding properties of SRSF7 Zn-knuckle domain and the control of FAS/CD95 alternative splicing.

18. Jakubauskiene E, Vilys L, Makino Y, et al. Increased SR protein phosphorylation changes pre-mRNA splicing in hypoxia. J Biol Chem 2015; May 28.

*19. Bordeleau F, Califano JP, Negron Abril YL, et al. Tissue stiffness regulates serine/arginine-rich protein-mediated splicing of the extra domain B-fibronectin isoform in tumors. Proc Natl Acad Sci U S A 2015; Jun 23.

This paper provides the first evidence that the stiffness of the extracellular matrix controls alternative splicing through regulation of SR proteins phosphorylation. These data unravel a mechanism by which tumor cells may respond and adapt to their mechanical microenvironment. 
20. Naro C, Bielli P, Pagliarini V, Sette C. The interplay between DNA damage response and RNA processing: the unexpected role of splicing factors as gatekeepers of genome stability. Front Genet 2015; 6:142.

**21. Tresini M, Warmerdam DO, Kolovos $\mathrm{P}$, et al. The core spliceosome as target and effector of non-canonical ATM signaling. Nature 2015; Jun 24.

Besides DNA double strand breaks and chromatin remodeling, this paper identifies a new way to activate ATM and defines a previously unknown mechanism by which ATM maintains genome stability.

**22. Colla S, Ong DS, Ogoti Y, et al. Telomere dysfunction drives aberrant hematopoietic differentiation and myelodysplastic syndrome. Cancer Cell 2015; 27:644-657.

This study describes an unpreviously known link between signaling from telomere erosion, aberrant RNA splicing and biased differentiation of myeloid progenitors to drive myeolodysplastic syndrome features. Interestingly, it identifies the DNA methyltransferase DNMT3A as a potential target of SRSF2 protein.

23. Matsuoka S, Ballif BA, Smogorzewska A, et al. ATM and ATR substrate analysis reveals extensive protein networks responsive to DNA damage. Science 2007; 316:11601166.

**24. Savage KI, Gorski JJ, Barros EM, et al. Identification of a BRCA1-mRNA splicing complex required for efficient DNA repair and maintenance of genomic stability. Mol Cell $2014 ; 54: 445-459$

This paper highlights BRCA1, a critical component of the DNA Damage Response (DDR), as a full partner of the spliceosome machinery involved in DNA damage-induced alternative splicing events. Splicing factors can also contribute to DNA repair mechanisms through their interaction with DDR components. By providing evidence that DDR components can also 
control alternative splicing, this study adds another level in the interplay between RNA splicing and DNA damage machineries.

25. Wojtuszkiewicz A, Assaraf YG, Maas MJ, et al. Pre-mRNA splicing in cancer: the relevance in oncogenesis, treatment and drug resistance. Expert Opin Drug Metab Toxicol $2014 ; 11: 673-689$.

26. Dehm SM. mRNA splicing variants: exploiting modularity to outwit cancer therapy. Cancer Res 2013; 73:5309-5314.

27. Shi H, Hugo W, Kong X, et al. Acquired resistance and clonal evolution in melanoma during BRAF inhibitor therapy. Cancer Discov 2014; 4:80-93.

28. Hsu TY, Simon LM, Neill NJ, et al. The spliceosome is a therapeutic vulnerability in MYC-driven cancer. Nature 2015; 525: 384-388.

29. Araki S, Dairiki R, Nakayama Y, et al. Inhibitors of CLK protein kinases suppress cell growth and induce apoptosis by modulating pre-mRNA splicing. PLoS One 2015; 10:e0116929.

30. Mavrou A, Brakspear K, Hamdollah-Zadeh M, et al. Serine-arginine protein kinase 1 (SRPK1) inhibition as a potential novel targeted therapeutic strategy in prostate cancer. Oncogene 2014; Nov 10.

**31. Wang P, Zhou Z, Hu A, et al. Both decreased and increased SRPK1 levels promote cancer by interfering with PHLPP-mediated dephosphorylation of Akt. Mol Cell 2014; $54: 378-391$.

This paper demonstrates that SRPK1 exerts dual functions, either oncogenic or tumor suppressive, through its interaction with the AKT phosphatase PHLPP and thus the control of AKT phosphorylation. SRPK1 pharmacological inhibitors are currently in clinical development and have been shown to exert anti-tumor functions in pre-clinical studies. This paper suggests that their effects might be deleterious depending on the tumor type. 
*32. van Roosmalen W, Le Devedec SE, Golani O, et al. Tumor cell migration screen identifies SRPK1 as breast cancer metastasis determinant. J Clin Invest 2015; 125:1648-1664. Through the use of a large scale siRNA screen devoid to the identification of genes affecting tumor cell migration, this paper identifies SRPK1 as a critical determinant of breast cancer metastasis.

33. Yoshida $\mathrm{T}$, Kim $\mathrm{JH}$, Carver $\mathrm{K}$, et al. CLK2 Is an Oncogenic Kinase and Splicing Regulator in Breast Cancer. Cancer Res 2015; 75:1516-1526.

*34. Convertini P, Shen M, Potter PM, et al. Sudemycin E influences alternative splicing and changes chromatin modifications. Nucleic Acids Res 2014; 42:4947-4961.

This paper adds further evidence of the link between pre-mRNA alternative and chromatin remodelling through the demonstration that the SF3B1 spliceosome inhibitor sudemycin E induces a loss of H3K36me3 histone mark in chromatin encoding alternatively spliced exons.

35. Naftelberg S, Schor IE, Ast G, Kornblihtt AR. Regulation of alternative splicing through coupling with transcription and chromatin structure. Annu Rev Biochem 2015; 84:165-198.

**36. Sanidas I, Polytarchou C, Hatziapostolou M, et al. Phosphoproteomics screen reveals akt isoform-specific signals linking RNA processing to lung cancer. Mol Cell 2014; 53:577590.

This paper provides the first evidence that chromatin-regulated RNA splicing machinery is under the control of AKT1/3 signals.

37. Xargay-Torrent S, Lopez-Guerra M, Rosich L, et al. The splicing modulator sudemycin induces a specific antitumor response and cooperates with ibrutinib in chronic lymphocytic leukemia. Oncotarget 2015; Jun 8.

**38. Salton M, Kasprzak WK, Voss T, et al. Inhibition of vemurafenib-resistant melanoma by interference with pre-mRNA splicing. Nat Commun 2015; 6:7103. 
This paper establishes proof-of-principle that splicing modulation can prevent the growth of melanoma cells with acquired resistance to vemurafenib owing to the expression of the BRAF3-9 splice variant devoid of its RAS-binding domain. It opens the way to the future development of new therapeutic strategies aiming at target the splicing machinery to overcome drug resistance.

39. Eskens FA, Ramos FJ, Burger $\mathrm{H}$, et al. Phase I pharmacokinetic and pharmacodynamic study of the first-in-class spliceosome inhibitor E7107 in patients with advanced solid tumors. Clin Cancer Res 2013; 19:6296-6304.

\section{Acknowledgements}

The authors were supported by the Comité Départemental Isère de la Ligue Nationale contre le Cancer, by INCa/Canceropôle Ile de France, by the Fondation pour la Recherche Médicale and by the Fondation ARC pour la Recherche Contre le Cancer. Cherine Abou Faycal is supported by the Ligue Nationale Contre Le Cancer.

\section{Figure 1 legend}

In tumors, alterations of core components of the spliceosome machinery that participate in all the steps of RNA splicing by binding to branch site, 3' splice or 5' splice site, or specific exonic sequences are illustrated. These alterations include mutations or deregulated expression. Concomittantly, various stressful stimuli can alter the expression and/or the activity of these proteins. This leads to aberrant splicing patterns in numerous target mRNAs, including intron retention or exon poison inclusion, which may reduce mRNA levels or generate truncated protein isoforms with defective functions. By this way, spliceosome alterations contribute to the hallmarks of cancer model proposed by Hanahan and Weinberg, leading to tumor progression as well as to tumor escape from therapy. Alterations of the 
spliceosome machinery and more generally global RNA splicing defects may render tumor cells sensitive to spliceosome inhibition, therefore providing an Achilles heel for future therapeutic strategies. ESE: Exonic Splicing Element; ISE: Intronic Splicing Element.

\section{Conflict of Interest}

The authors declare no conflict of interest. 